\title{
Effect of surfactants on the rate of growth of an air bubble by rectified diffusion
}

\author{
Judy Lee ${ }^{1, *}$, Sandra Kentish ${ }^{1, *}$ and Muthupandian Ashokkumar ${ }^{2, *}$ \\ *email: masho@unimelb.edu.auＦax:+61393475180
}

\section{Affiliations:}

* Particulate Fluids Processing Centre, ${ }^{1}$ Department of Chemical and Biomolecular Engineering, ${ }^{2}$ School of Chemistry, University of Melbourne, VIC 3010, Australia.

\begin{abstract}
The rectified diffusion growth of a single air bubble levitated in an acoustic field (frequency $=22.35 \mathrm{kHz}$ ) in water and in aqueous solutions containing surfactants (sodium dodecyl sulphate and sodium dodecylbenzene sulfonate) was investigated. As reported by Crum (J. Acoust. Soc. Am. 68 (1980) 203), the presence of surfactants at the bubble/liquid interface enhanced the growth rate of the bubble by rectified diffusion. It is suggested in this paper that in addition to the effect of surfactants on the surface tension and interfacial resistance to mass transfer, the effect of surface rheological properties may also contribute to the cause of the enhancement observed in the bubble growth rate.
\end{abstract}




\section{Introduction}

Acoustic cavitation refers to the ultrasound-induced formation, growth and collapse of microbubbles in a liquid. The growth of these microbubbles occurs by a process known as rectified diffusion. This happens when the acoustic pressure is above a certain threshold. The growth of a bubble by rectified diffusion results from the uneven mass transfer rate across the air-liquid interface ${ }^{1}$. This uneven mass transfer was explained in terms of the 'area' and the 'shell' effects by Crum ${ }^{1}$.

There have been numerous attempts to model the acoustic pressure threshold for rectified diffusion. Blake ${ }^{2}$ was the first to develop theoretical equations describing rectified diffusion. However, Strasberg ${ }^{3}$ later experimentally measured the sound pressure for rectified diffusion and observed values which were six times smaller than Blake's predicted threshold values. It was argued that the cause of this discrepancy was the effects of convection and bubble wall motions that were not accounted for. An improved theoretical model that included these effects was presented by Hsieh and Plesset ${ }^{4}$. Their theory provided a better agreement with Strasberg's ${ }^{5}$ experimental values. It was initially thought that Hsieh and Plesset's theory was restricted to small sinusoidal oscillations and this led Eller and Flynn ${ }^{6}$ to develop another theory to account for large sinusoidal oscillations. However, Safar ${ }^{7}$ demonstrated that when the inertial terms in Hsieh and Plesset's theory were not neglected, the same results were obtained from the theory developed by Eller and Flynn. 
Although the threshold for rectified diffusion can be adequately predicted by theory, Eller ${ }^{8,9}$ found that the above mentioned theory underestimated the growth rate above the rectified diffusion threshold. Eller alluded to the possibility that acoustic streaming around the bubble may have increased the mass transport rate, resulting in the enhancement of the bubble growth rate. Experimental limitations prevented Eller from observing whether the onset of surface oscillations had occurred during his measurements. Surface oscillations were detected by Gould ${ }^{10}$ and they were believed to be responsible for the acoustic streaming around the bubble, enhancing bubble growth rates. It was also shown that when acoustic streaming was absent the measured growth rates agreed well with theory. Crum ${ }^{1}$ then refined the theory to account for the thermodynamic behaviour of the bubble interior and demonstrated a strong agreement between the theoretically predicted and experimentally measured values of threshold and growth rate. However, discrepancies were evident with the addition of a surfactant. It was found that even before the onset of surface oscillations the presence of the surfactant decreased the threshold and enhanced the growth rate substantially. Clearly the surface tension term in the theory was inadequate in accounting for the effect of the surfactant. Crum gave several plausible causes for this anomaly the most probable cause being that the presence of the surfactant may possibly have induced acoustic streaming even in the absence of surface oscillations ${ }^{1}$.

Fyrillas and Szeri ${ }^{11}$ put forward a new theoretical analysis to describe the mass transportation in the process of rectified diffusion. In an effort to explain the enhancement in the bubble growth rate Fyrillas and Szeri ${ }^{12,13}$ later extended their analysis to incorporate the effect of interfacial resistance to mass transfer caused by 
the surfactant molecules at the interface. Using the interfacial resistance data obtained by Caskey and Barlage ${ }^{14}$, Fyrillas and Szeri ${ }^{12}$ examined the effect of three surfactants on the rate of bubble growth. They demonstrated that depending upon how the interfacial resistance varied as a function of the surfactant surface concentration, the mass transport may be enhanced or retarded. Crum had used a commercial surfactant Photoflo for which the adsorption isotherm and interfacial resistance data were unavailable, therefore direct confirmation of Fyrillas and Szeri’s theory and Crum's results was not possible ${ }^{12}$.

Crum's work ${ }^{1}$ remains the only experimental report of the enhanced growth rate of an air bubble by rectified diffusion in the presence of a surfactant. However, the understanding of the bubble dynamics in the presence of surfactants is crucial for some applications such as ultrasound contrast agents ${ }^{15,16}$. Therefore, in this investigation a bubble undergoing rectified diffusion growth in the absence and in the presence of surfactants Sodium dodecyl sulfate (SDS) and Sodium dodecyl benzene sulfonate (SDBS) were performed.

\section{Experimental Details}

The surfactants used were high purity research grade samples. SDS was from BDH and SDBS was from Sigma Aldrich. The desired surfactant concentration was made by diluting a stock surfactant solution of $0.1 \mathrm{M}$ in Milli-Q water (conductivity less than $10^{-6} \mathrm{~S} / \mathrm{cm}$ at $21^{\circ} \mathrm{C}$ ). These solutions were left overnight to allow complete air saturation and equilibrium to be reached before they were used. Air saturated water ensured that the air concentration remained constant throughout the course of the 
experiment and that reproducible results were obtained. The temperature throughout the duration of the experiment did not change by more than $0.4{ }^{\circ} \mathrm{C}$.

The single bubble experimental setup employed was similar to that described by Ashokkumar and Grieser ${ }^{17}$ and Matula ${ }^{18}$. The single bubble levitation cell used was a cylindrical pyrex cell with a diameter of $6.5 \mathrm{~cm}$ and a height of $11.5 \mathrm{~cm}$. The cell had two parallel flat surfaces for viewing and backlighting purposes. Attached at the bottom of this cell (with the aid of an epoxy resin (Araldite)) was a hollow cylindrical Lead Zirconate Titanate (PZT) piezo ceramic transducer, obtained from the American Piezo Ceramics Inc. The transducer was driven by a programmable $15 \mathrm{MHz}$ Hameg function generator (HM8131-2) at a resonance frequency of $22.35 \mathrm{kHz}$ via a KrohnHite power amplifier (Model 7500). The function generator also supplied another output signal to the stroboscope (Model 610/1581) that drove a light emitting diode (LED) in synchronization with the ultrasound frequency. This strobe technique, described by Tian et al. ${ }^{19}$ in detail, enabled a bubble at a given point within an acoustic cycle to be imaged. For practical reasons, the strobe was adjusted to capture the bubble at the maximum size during its radial oscillations.

A single bubble was introduced with the aid of a syringe. Once the bubble was trapped at the antinode, the bubble image was magnified using a Specwell (10 x 30, extra short focus) microscope, recorded using a DAG MTI CCD100 video camera and sent to a PC via a Matrox Meteor video capture card. The magnification of the imaging system was determined by taking an image of a needle of a known diameter. From this, the diameter of the bubbles was determined. 
All experiments were conducted at a constant acoustic pressure of 0.22 bar. This acoustic pressure was measured using an interchangeable $1 \mathrm{~mm}$ PVdF calibrated needle hydrophone (Precision Acoustics Ltd). The signal from the needle hydrophone was then relayed to a Tektronix digital oscilloscope (model TDS360) with a termination impedance of 50 ohms.

\section{Results}

Once a bubble below the resonance size and above the threshold for rectified diffusion was captured at the antinode, the bubble remained very stable as it grew. As the bubble size increased, the buoyancy force increased and this led to an observation of a small upward displacement in the bubble position. Figure 1 shows a series of images of a single levitated bubble undergoing rectified diffusion in the presence of $2.4 \mathrm{mM}$ of SDS. The last frame shows the bubble undergoing volume oscillations as it approaches the resonance size. This volume oscillation was accompanied by large bubble instabilities and often resulted in the removal or break up of the bubble.

The change in size of bubbles with initial radii of both $35 \mu \mathrm{m}$ and $22 \mu \mathrm{m}$ in water as a function of time is shown in Figure 2. For a $35 \mu \mathrm{m}$ bubble three repeated runs are included to demonstrate the reproducibility of the experiments which had an average error of approximately $\pm 5 \%$. This error increases towards the resonance size. For a $20 \mu \mathrm{m}$ bubble, the bubble size remains constant over the period of 300 seconds and then slowly dissolves. This shows that $20 \mu \mathrm{m}$ is below the threshold bubble radius for rectified diffusion to take place at an acoustic pressure of 0.22 bar and a frequency of $22.35 \mathrm{kHz}$. Crum has indicated that a threshold bubble radius of $32 \mu \mathrm{m}$ at $0.22 \mathrm{bar}$ and $22.1 \mathrm{kHz}^{1}$ is needed for rectified diffusion to occur. 
Figure 3 shows the bubble radii as a function of time for three different bulk concentrations of SDS. Note that the initial radii of the bubbles were about $25 \mu \mathrm{m}$, which is smaller than the threshold radius mentioned above. It has been suggested in an earlier report ${ }^{1}$ that the threshold radius for rectified diffusion is lowered in the presence of a surfactant. As the bubble approaches the resonance size (before the onset of volume oscillations) the growth rate appears to increase. The circles in the plots (Figure 3) denote the observation of volume oscillations. This figure shows that there appears to be a slight increase in the bubble growth rate with an increase in the SDS bulk concentration.

The bubble growth rate as a function of the bulk concentration of SDS, calculated by measuring the slope on the radius time curve at a bubble radius of $40 \mu \mathrm{m}$ (extracted from Figure 2 and Figure 3), is presented in Figure 4. This figure shows that for a given acoustic pressure and bubble radius, the rate of bubble growth increases linearly with increasing SDS bulk concentration. The bubble growth rate (an average of 3 independent measurements) for $3 \mathrm{mM}$ of SDBS is also shown in the figure. Using a pendant drop tensiometer the surface tension for SDS and SDBS at this bulk concentration was measured and found to be $48 \mathrm{dyn} / \mathrm{cm}$ and $35 \mathrm{dyn} / \mathrm{cm}$, respectively. Despite the dissimilarities in the surface activity, the growth rate of the bubble for SDBS does not vary significantly from that of SDS at this bulk concentration. 


\section{Discussion}

The results presented for surfactant systems reinforce the observations of Crum ${ }^{1}$ : the presence of a surfactant indeed enhances the rate of growth of a bubble undergoing rectified diffusion compared to that in the absence of a surfactant. Crum ${ }^{1}$ had plotted the bubble growth rate for Photoflo as a function of surface tension. A similar plot for SDS, SDBS is shown in Figure 5. The experimental data for Photoflo and the theoretical equation developed by Crum ${ }^{1}$ are also included . The bubble growth rate obtained by Crum was for a bubble radius of $50 \mu \mathrm{m}$ whereas the growth rate obtained in this study was for a bubble radius of $40 \mu \mathrm{m}$. Owing to the volume oscillations occurring at a bubble radius of approximately $50 \mu \mathrm{m}$ in the presence of SDS the growth rate at this radius could not be obtained.

Figure 5 shows that the growth rate for an air bubble in water observed in our experiment is higher than that obtained by Crum ${ }^{1}$. The growth rate for water was not shown by Crum ${ }^{1}$, however, the rate at $68 \mathrm{dyn} / \mathrm{cm}$ would be approximately $1 \mu \mathrm{m} / 100 \mathrm{~s}$. The difference between the growth rates would be even greater if Crum had chosen a bubble radius of $40 \mu \mathrm{m}$ instead of a $50 \mu \mathrm{m}$ bubble.

A possible cause of the discrepancy is the use of different experimental conditions. Crum ${ }^{1}$ measured the bubble size by measuring the rise velocity of the bubble when the sound field is turned off. An error of $10 \%$ in the bubble radius is associated with the rise velocity measurements. Bubble dissolution during the rise time would increase the error further. Crum also reported a surface tension of $68 \mathrm{dyn} / \mathrm{cm}$ for water, whereas in our study, a surface tension of $72 \mathrm{dyn} / \mathrm{cm}$ was obtained. This apparent difference in water purity may also account for differences in experimental 
results. Finally, Crum measured the growth in equilibrium bubble size, whereas we report the growth in maximum bubble size during the radial oscillations.

Figure 5, shows that for all three surfactant systems, the growth rate increases with decreasing surface tension. However, for a given surface tension, these growth rates are different. The surface tension plotted in Figure 5 is the equilibrium surface tension where equilibrium adsorption is assumed. However, for an oscillating bubble surface, equilibrium adsorption may not be achieved and it may not be appropriate to compare different surfactants as a function of surface tension. A better approach would be to plot the growth rate as a function of moles of solute for a given volume as shown in Figure 4. This is not possible for Photoflo because the surface activity and concentration of Photoflo were not specified. It was reported by Fyrillas and Szeri ${ }^{12}$ that in addition to the orthoxylated octylphenol in Photoflo there are other unspecified components present. The observed similarities in bubble growth rates between SDS and SDBS in Figure 4 suggest that, in a dynamic situation, the concentration of adsorbed surfactant molecules may be of the same level. A similar observation has been reported in a sonoluminescence study involving surfactants with different surface activity ${ }^{20-22}$. The extent to which the sonoluminescence was influenced did not depend upon the surface concentrations of the surfactants.

Figure 5 shows that the theory presented by Crum $^{1}$ is inadequate in estimating the bubble growth rate with decreasing surface tension due to the addition of a surfactant. As discussed in the introduction, different theories on the effect of surfactants on the bubble growth via rectified diffusion have been presented in the literature ${ }^{1,12,13}$. Crum 
had stated that a likely scenario is that of acoustic streaming in the absence of volume oscillation.

Alternatively, Fyrillas and Szeri ${ }^{12,13}$ presented a theoretical analysis to include the interfacial resistance to mass transfer term in the presence of a surfactant and demonstrated that surfactants can enhance and retard bubble growth rate. Again the growth rates obtained in this present study appear to be higher than predicted by this model. Exact comparison is difficult without repeating the complex mathematical analysis.

The presence of surfactants may also cause changes in the rheological properties of the bubble surface ${ }^{23-26}$. At SDS concentrations below the CMC, (approximately $8 \mathrm{mM}$ ), the bulk viscosity is not affected ${ }^{27}$. However, at these low SDS bulk concentrations it has been shown that the surface exhibits both viscous and elastic (viscoelastic) properties ${ }^{23,24,28,29}$.

The surface viscoelastic properties have been shown to be a function of both the bulk concentration of surfactant and the oscillation frequency ${ }^{23,24,28,29}$. There is no known work to the authors' knowledge on the surface viscoelastic properties in the presence of SDS at a frequency in the kilohertz range. However, at frequencies below $500 \mathrm{~Hz}$ there is evidence that with increasing SDS concentration up until $3 \mathrm{mM}$ there is a measurable increase in the surface viscosity and elasticity ${ }^{23,24,28,29}$. Kloek et al. ${ }^{30}$ have theoretically demonstrated that both surface viscosity and elasticity can retard the bubble dissolution process, indeed the latter can actually stop the dissolution completely. They have shown experimentally that surface properties of a glycinin film 
slowed down the bubble shrinkage rate ${ }^{31}$. Ronteltap and Prins ${ }^{32}$ have also shown experimentally that a bubble shrinks more slowly in a solution with a high surface viscosity .

It is unknown whether the surface viscoelastic properties can enhance bubble growth rate as well. The results appear to indicate a correlation between the enhancement in the bubble growth rate and the increase in the bubble's surface viscoelastic properties when the concentration of SDS is increased.

\section{Conclusions}

In summary, the present study shows that the increase in the bubble growth rate as a function of bulk concentrations of SDS and SDBS compliments the result reported by Crum ${ }^{1}$ for photoflo. There remains the need for detailed modelling to validate current mathematical models, which include both surface tension and interfacial resistance term, with the experimental results obtained in this study. However, another possible explanation for the increase in the rectified diffusion growth in the presence of surfactants may be the surface rheological effects. It has been demonstrated theoretically in the literature that surface elasticity and viscosity can affect mass transfer across the bubble/solution interface. There appears to be a correlation between an increase in surfactant concentration and an increase in the surface rheological properties. 


\section{Acknowledgments}

The authors would like to acknowledge Prof. Franz Grieser for his valuable comments and suggestions. The financial support from Kodak and from the Particulate Fluids Processing Centre, a Special Research Centre of the Australian Research Council, is also acknowledged. Judy Lee receives a postgraduate stipend from the Department of Chemical and Biomolecular Engineering within the University of Melbourne and this support is also gratefully acknowledged. 


\section{References}

(1) Crum, L. A. J. Acoust. Soc. Am. 1980, 68, 203.

(2) Blake Jr., F. G. Tech. Memo. No. 12, Acoust. Res. Lab.Harvard Uni., U.S.A. 1949.

(3) Strasberg, M. J. Acoust. Soc. Am. 1959, 31, 163.

(4) Hsieh, D. Y.; Plesset, M. S. J. Acoust. Soc. Am. 1961, 33, 206.

(5) Strasberg, M. J. Acoust. Soc. Am. 1961, 33, 359.

(6) Eller, A. I.; Flynn, H. G. J. Acoust. Soc. Am. 1965, 37, 493.

(7) Safar, M. H. J. Acoust. Soc. Am. 1968, 43, 1188.

(8) Eller, A. I. J. Acoust. Soc. Am. 1969, 46, 1246.

(9) Eller, A. I. J. Acoust. Soc. Am. 1972, 52, 1447.

(10) Gould, R. K. J. Acoust. Soc. Am. 1974, 56, 1740.

(11) Fyrillas, M. M.; Szeri, A. J. J. Fluid Mech. 1994, 277, 381.

(12) Fyrillas, M. M.; Szeri, A. J. J. Fluid Mech. 1995, 289, 295.

(13) Fyrillas, M. M.; Szeri, A. J. J. Fluid Mech. 1996, 311, 361.

(14) Caskey, J. A.; Barlage, W. B. J. Colloid Interface Sci. 1972, 41, 52.

(15) Chen, W. S.; Matula, T. J.; Crum, L. A. Ultrasound in Med. \& Biol. 2002, 28, 793.

(16) Chen, W. S.; Brayman, A. A.; Matula, T. J.; Crum, L. A.; Miller, M. W. Ultrasound in Med. \& Biol. 2003, 29, 739.

(17) Ashokkumar, M.; Grieser, F. J. Am. Chem. Soc. 2000, 122, 12001.

(18) Matula, T. J. Phil. Trans. R. Soc. Lond. A 1999, 357, 225.

(19) Tian, Y.; Ketterling, J. A.; Apfel, R. E. J. Acoust. Soc. Am. 1996, 100, 3976.

(20) Ashokkumar, M.; Hall, R.; Mulvaney, P.; Grieser, F. J. Phys. Chem. B 1997, 101, 10845.

(21) Sostaric, J. Z.; Riesz, P. J. Phys. Chem. B 2002, 106, 12537.

(22) Sostaric, J. Z. Interfacial effects on aqueous sonochemistry and sonoluminescence. Ph.D. Thesis, The University of Melbourne, 1999.

(23) Kao, R. L.; Edwards, D. A.; Wasan, D. T.; Chen, E. J. Colloid Interface Sci. 1992, 148, 247.

(24) Wantke, K. D.; Fruhner, H.; Ortegren, J. Colloid Surf. A 2003, 221, 185.

(25) Warburton, B. Interfacial rheology. In Rheological measurement; Collyer, A. A., Clegg, D. W., Eds.; Chapman \& Hall: London, 1998; pp 723.

(26) Danov, K. D.; Kralchevsky, P. A.; Ivanov, I. B. Equilibrium and dynamics of surfactant adsorption monlayers and thin liquid films. In Handbook of detergents. Part A: properties; Broze, G., Ed.; Marcel Dekker: New York, 1999; Vol. 82; pp 303.

(27) Greener, J.; Contestable, B. A.; Bale, M. D. Macromolecules 1987, 20, 2490.

(28) Djabbarah, N. F.; Wasan, D. T. Chem. Eng. Sci. 1982, 37, 175.

(29) Tian, Y.; Holt, R. G.; Apfel, R. E. J. Colloid Interface Sci. 1997, 197, 1.

(30) Kloek, W.; Van Vliet, T.; Meinders, M. J. Colloid Interface Sci. 2001, 237, 158.

(31) Meinders, M. B. J.; Bos, M. A.; Lichtendonk, W. J.; van Vliet, T. Effects of stress relaxation in soy glycinin films on bubble dissolution and foam stability. In Food Colloids, Biopolymers and Materials; Dickinson, E., van Vliet, T., Eds.; Royal Society of Chemistry: Cambridge, 2003; pp 156.

(32) Ronteltap, A. D.; Prins, A. Colloids Surfaces 1990, 47, 285. 
Figure 1: Rectified diffusion growth of a single levitated bubble in the presence of $2.4 \mathrm{mM}$ of SDS at an acoustic pressure of $0.22 \pm 0.03$ bar. The number in the figure denotes the frame number (duration between frames is 30 seconds).

Figure 2: The variation in bubble radius as a function of time in air saturated water at $0.22 \pm 0.03$ bar acoustic pressure for an initial bubble radius of $22 \mu \mathrm{m}$ and $35 \mu \mathrm{m}$.

Figure 3: The variation in the bubble radius as a function of time for different bulk concentrations of SDS for bubbles with initial radii of $25 \mu \mathrm{m}$ and at an acoustic pressure of $0.22 \pm 0.03$ bar. The circled data indicates when volume oscillations were imaged.

Figure 4: Bubble growth rate as a function of SDS and SDBS bulk concentrations for a $40 \mu \mathrm{m}$ bubble at an acoustic pressure of $0.22 \pm 0.03$ bar.

Figure 5: Bubble growth rate vs. surface tension for SDS, SDBS and Photoflo solutions. The solid line is the theoretical line calculated from Eq. (13) in reference ${ }^{1}$. For SDS and SDBS the bubble growth rate was calculated at a bubble radius of $40 \mu \mathrm{m}$ and for Photoflo and the theoretical line (obtained by Crum ${ }^{1}$ ) a bubble radius of $50 \mu \mathrm{m}$ was used. 
Figure 1

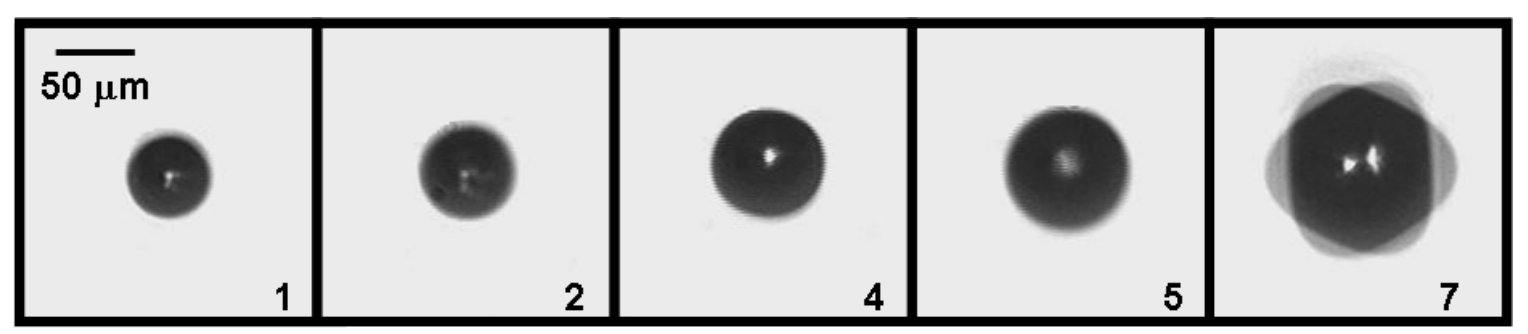


Figure 2

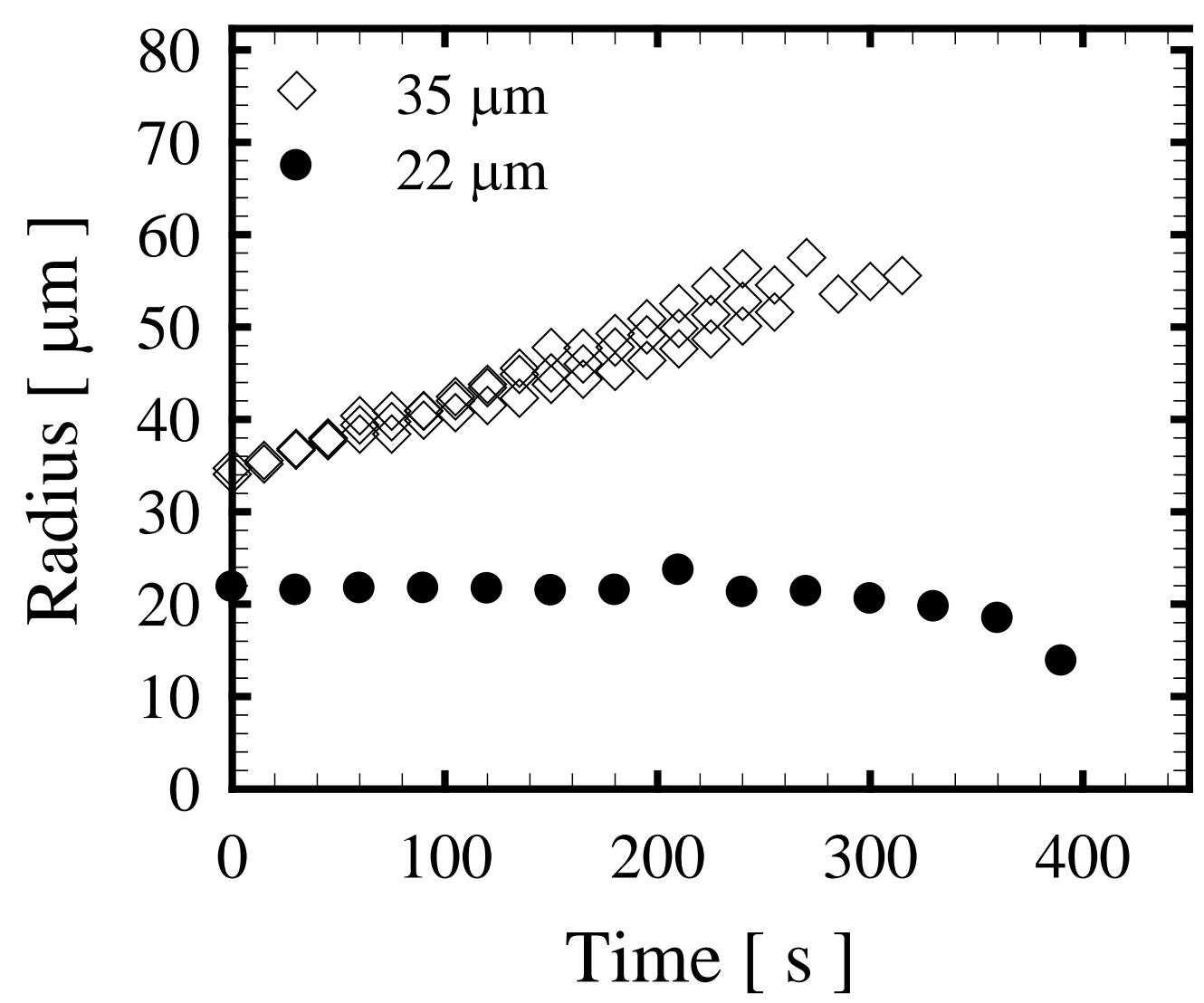


Figure 3

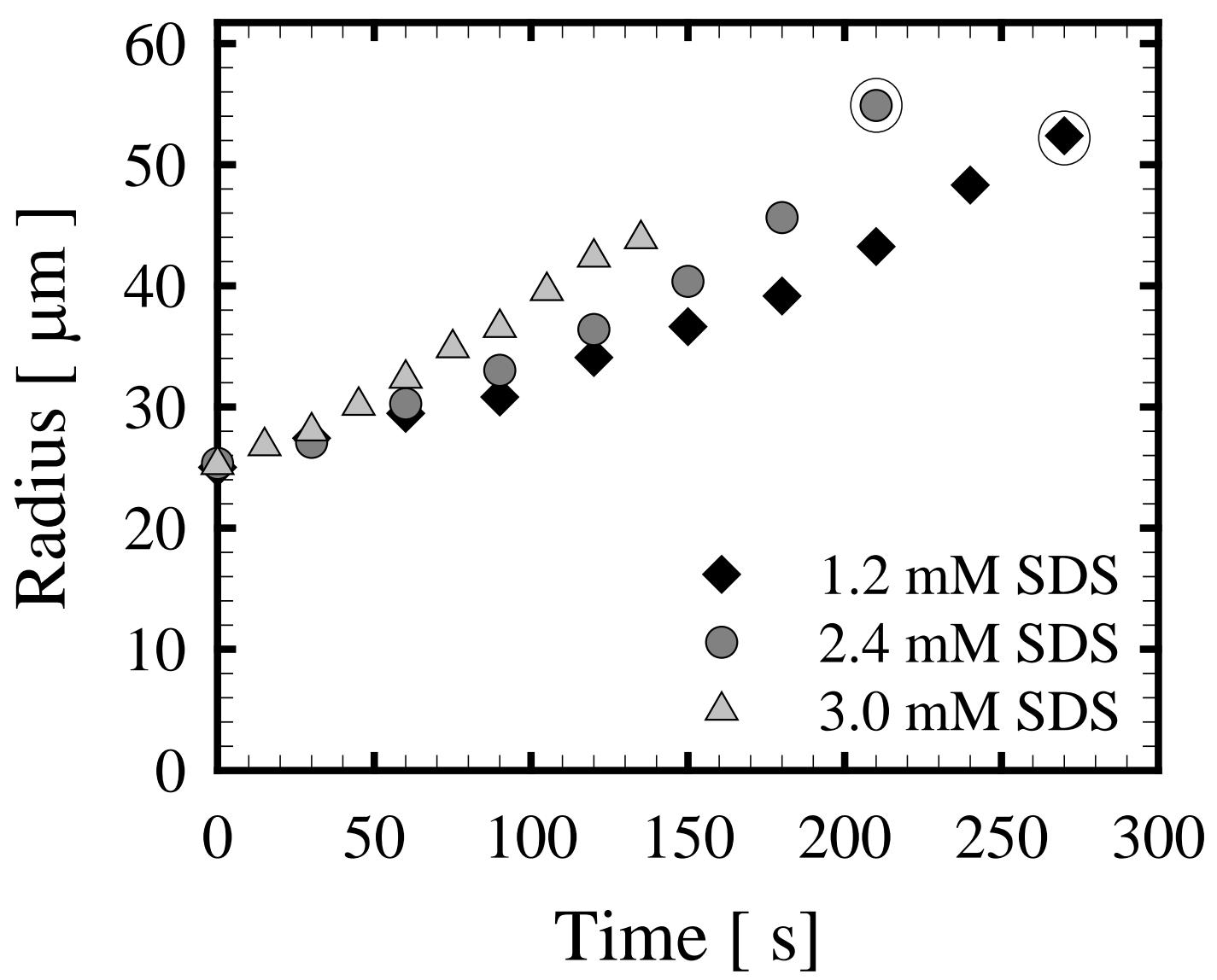


Figure 4

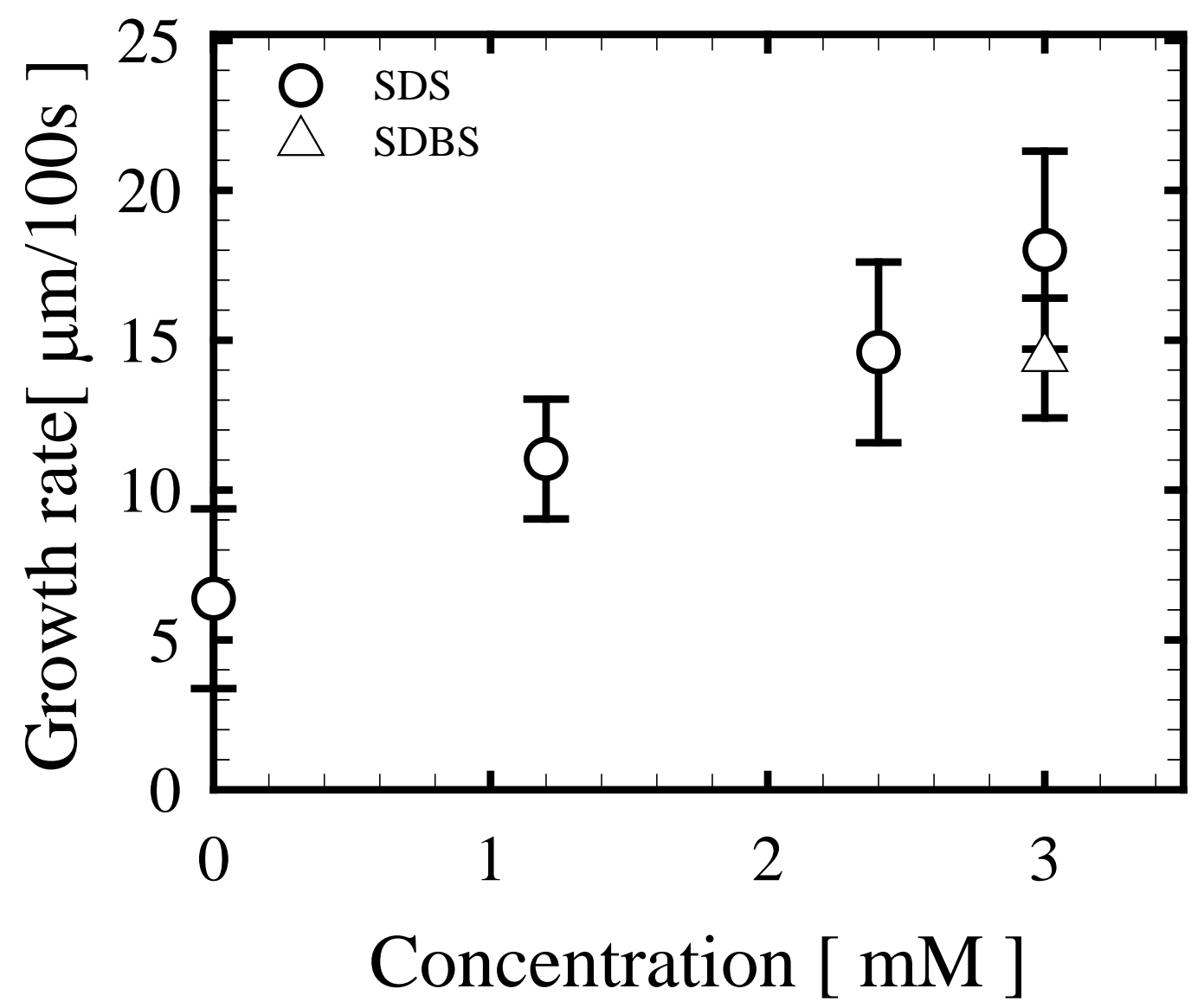


Figure 5

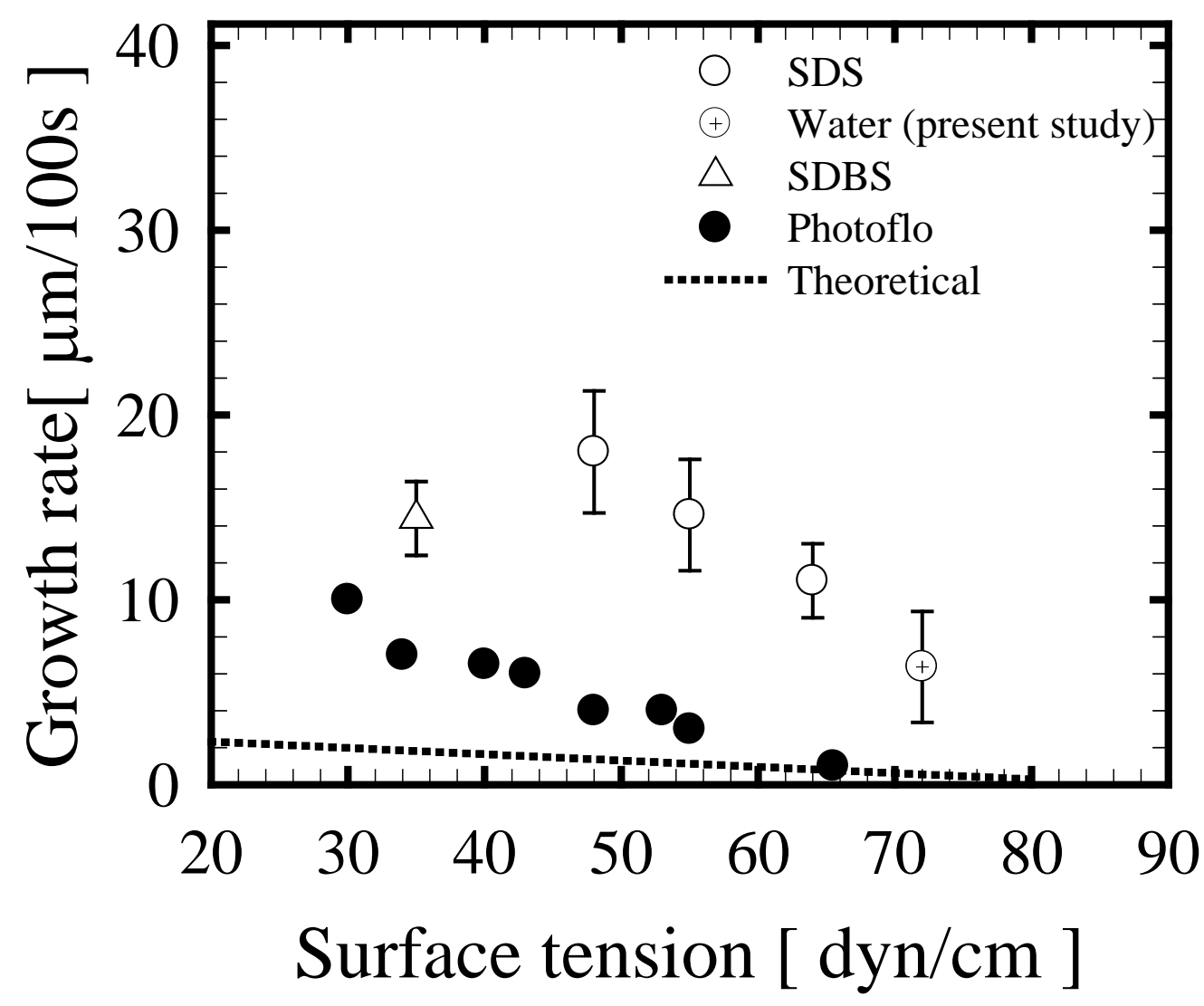

